\title{
Transformar la asistencia de necesidad básica en un momento de cuidado*
}

\author{
Transform Basic Need Assistance at a Time of Care \\ Transformar a assistência de necessidade básica em um momento de cuidado
}

Ligia Patricia Arroyo Marlés

Universidad de La Sabana, Colombia

DOI: https://doi.org/10.11144/Javeriana.ie22.tanb

ORCID: https://orcid.org/0000-0002-4317-6722

Recibido: 17 Julio 2019

Beatriz Pérez Giraldo ${ }^{a}$

Universidad de La Sabana, Colombia

Aceptado: 10 Junio 2020

beatriz.perez@unisabana.edu.co

ORCID: https://orcid.org/0000-0001-6636-290X

Publicado: 29 Diciembre 2020

Mónica Andrea Nonsoque Cholo

Universidad de La Sabana, Colombia

ORCID: https://orcid.org/0000-0001-6475-4854

Beatriz Sánchez Herrera

Universidad de La Sabana, Colombia

ORCID: https://orcid.org/0000-0002-8029-7187

Maryory Guevara Lozano

Universidad de La Sabana, Colombia

ORCID: https://orcid.org/0000-0002-9559-5697

\section{Resumen:}

Introducción. Conocer y atender debidamente las necesidades de un ser humano en medio de su vulnerabilidad continúa siendo un reto vigente. Objetivo. Describir la transformación de la "Atención a las necesidades básicas" en un momento de cuidado de enfermería para favorecer la adaptación del paciente y su cuidador familiar durante la hospitalización, en la Clínica Universidad de La Sabana, para el periodo 2018-2019. Método. Esta es una investigación metodológica en enfermería, realizada bajo lineamientos del Modelo de Enfermería Universidad de La Sabana, que incluyó cuatro fases. Resultados. Es preciso que la atención a las necesidades básicas sea respaldada en la mejor evidencia disponible, y con un trato humanizado. Hacer visible este proceso permitió medirlo y mejorarlo. Conclusiones. Para brindar un cuidado humano y seguro, no basta la buena intención, es necesario garantizar dos condiciones; por un lado, el compromiso requerido para soportarlo con la mejor evidencia disponible, aumentando la calidad del cuidado y la calidez o tono humano; y, por otro, el respeto y reconocimiento de la dignidad de las personas, favoreciendo el vínculo con ellas y su autonomía, lo que a su vez repercute positivamente en el desarrollo de la enfermería.

Palabras clave: investigación metodológica en enfermería, adaptación, evaluación de necesidades, atención de enfermería, teoría de enfermería.

\begin{abstract}
:
Introduction. Knowing and duly attending to the needs of a human being in the midst of his vulnerability, continues to be an ongoing challenge. Objective. Describe the transformation of "Attention to basic needs" in a moment of nursing care to favor the adaptation of the patient and their family caregiver during hospitalization, Universidad de La Sabana Clinic, 2018-2019. Method. This is a Nursing Methodology Research under the guidelines of the University of La Sabana Nursing Model that included four phases. Results. It is necessary that the attention to basic needs is supported by the best available evidence and in the human care. The description of this process helped the researchers to measure and improve it. Conclusions. To give humane and safe care, good intention is not enough, it is necessary to guarantee two conditions; the commitment required to support it in the best available evidence, improving the quality of care and the warmth or human tone; with respect and recognition of the dignity of people, favoring the bond with them, such as their autonomy, which in turn has a positive impact on the development of nursing.
\end{abstract}

Notas de autor

a Autora de correspondencia. Correo electrónico: beatriz.perez@unisabana.edu.co 
Keywords: nursing methodology research patient comfort, needs assessment, nursing care, nursing theory.

Resumo:

Introdução. Conhecer e atender devidamente as necessidades de um ser humano em meio à sua vulnerabilidade continua a ser um desafio atual. Objetivo. Descrever a transformação da "Atenção às necessidades básicas" num momento de cuidado de enfermagem para favorecer a adaptação do paciente e seu familiar cuidador durante a internação, na Clínica Universidad de La Sabana, para o período 2018-2019. Método. Trata-se de uma pesquisa metodológica em enfermagem, efetivada segundo diretrizes do Modelo de Enfermagem Universidade de La Sabana, que incluiu quatro fases. Resultados. É preciso que a atenção às necessidades básicas este amparada nas melhores evidências disponíveis e com um trato humanizado. Fazer visível esse processo permitiu medi-lo e aprimorálo. Conclusões. Para prestar um cuidado humano e seguro não bastam boas intenções, é preciso garantir duas condições; de um lado, o compromisso necessário para sustê-lo com as melhores evidências disponíveis, aumentando a qualidade do cuidado e o calor ou tom humano; e, de outro, respeito e reconhecimento da dignidade das pessoas, favorecendo o vínculo com elas e sua autonomia, o que por sua vez tem impacto positivo no desenvolvimento da enfermagem.

Palavras-chave: pesquisa metodológica em enfermagem, adaptação, avaliação de necessidades, atenção de enfermagem, teoria de enfermagem.

\section{Introducción}

Para la enfermería, el esquema de las necesidades básicas es parte de su historia, con importantes teoristas como Virginia Henderson, Faye Abdella y Dorotea Orem, quienes fundamentan sus planteamientos en los de Maslow (1). Sin embargo, conocer y atender debidamente las necesidades de un ser humano en medio de su vulnerabilidad continúa siendo un reto vigente, relacionado con la razón de ser de la profesión y la responsabilidad de la calidad y la humanización que pretenden los servicios de salud, y en particular el cuidado de enfermería (2).

El presente trabajo buscó describir el proceso llevado a cabo en la Clínica Universidad de La Sabana para cambiar una rutina de enfermería de "Atención a las necesidades básicas" y transformarla en un momento de cuidado de enfermería que favorezca la adaptación del paciente hospitalizado y su cuidador familiar.

\section{Método}

Investigación metodológica en enfermería que siguió los pasos propuestos por Polit y Tatano (3), desarrollada en alianza docencia-asistencia, en el marco del Campus Biomédico, bajo lineamientos del Modelo de Enfermería Universidad de La Sabana (4), con previo aval institucional. Tuvo en cuenta los parámetros éticos para investigación con seres humanos (5) y los lineamientos de protección ambiental de la institución (6). Atendiendo el método seleccionado, el estudio se desarrolló en cinco fases.

La primera consistió en una revisión de la literatura, que atendió lineamientos de Christmals y Gross (7), bajo la fórmula y descriptores [(Enfermería OR Atención de enfermería) AND (Necesidades humanas básicas OR Necesidades humanas)], en idiomas español, inglés y portugués, con una ventana de tiempo de dos años, sin límites geográficos, incluyendo solo publicaciones arbitradas. Se sumaron, posteriormente, referencias sugeridas por los autores revisados. El nivel de evidencia se clasificó bajo parámetros de Lobiondo y Haber, siendo 1 la máxima y 7 la mínima (8).

La segunda fase fue un diagnóstico del nivel de adaptación de los pacientes y sus cuidadores familiares durante la atención a las necesidades básicas. Este se realizó durante cuatro meses, referidos por los pacientes y familiares, los valorados por enfermería, el nivel de percepción de la satisfacción frente a la respuesta de enfermería a estas necesidades, los lineamientos institucionales, el nivel de adherencia de enfermería durante la atención, y el registro de la atención suministrada y su efecto. 
La tercera fase fue un planteamiento teórico de la transformación deseada con base el modelo institucional, y realizado a partir del diagnóstico y la revisión de las mejores prácticas mundiales, buscando que esta atención sea un verdadero momento de cuidado (9).

La cuarta fue la validación y ajuste a la propuesta de atención a necesidades básicas. Esta fue revisada por el grupo de la subdirección de enfermería, y socializada con el equipo interdisciplinario para hacerle los ajustes requeridos.

Finalmente, se tomaron los indicadores del Modelo de Enfermería Universidad de La Sabana para revisar la estructura, proceso y resultado de la transformación del momento frente al paciente o cuidador Familiar "N. ${ }^{\circ}$ pacientes o cuidadores que perciben adecuado cuidado de enfermería durante la asistencia a necesidades básicas / N. ${ }^{\circ}$ total de pacientes asistidos por enfermería y encuestados x 100”. Y para la enfermera es “N.․ de registros de atención a necesidades básicas por enfermería correctamente diligenciados en la historia clínica del paciente / N.. de registros de pacientes atendidos y auditados en sus necesidades básicas por enfermería x 100". De manera que se verifica si es un momento de cuidado. Se realizó un seguimiento por 2 años con los pacientes, cuidadores familiares y los enfermeros(as) en el servicio de hospitalización, que cuenta con 88 camas.

\section{Resultados}

\section{Fase I}

Primero, la literatura reporta 25 estudios, 3 de Asia, 6 de Europa, y 16 de América, con un importante aporte de América Latina. Sin embargo, el nivel de evidencia de estos estudios es débil, sin estudios de nivel 1 o 2; 1 de nivel 3; 6 de nivel 4; 3 de nivel 5; 11 de nivel 6; y 4 de nivel 7. Desde la perspectiva conceptual se puede establecer que las principales teorías de enfermería tienen origen cercano a las teorías de las necesidades humanas o se soportan en aquellas que las han considerado (1). Estas encaminan el cuidado para mejorar la práctica y responder de la mejor forma a las necesidades del paciente; es así como el plan de atención de enfermería, que incluye la valoración, diagnóstico, intervención y evaluación, permite dar una mirada integral a dichas necesidades y a los cuidados requeridos, y responde al objetivo de enfermería de fomentar la autonomía del paciente, para que se pueda integrar activamente a su entorno y la sociedad (10). De esta manera, atender las necesidades básicas es vital para mantener la salud y calidad de vida de las personas cuidadas, y la forma en que estas se atiendan es un determinante del desempeño enfermero (11).

La situación de hospitalización genera en los pacientes y familias un estado de vulnerabilidad que se suma al de la enfermedad, y tiende a desequilibrar la capacidad de satisfacer sus necesidades básicas; en tal sentido, valorarlas y atenderlas es una prioridad (12). Para ello, es necesario contar con un enfoque holístico que permita contemplar al sujeto de cuidado en su plenitud como ser humano (13). Un buen cuidado básico de enfermería es un pilar fundamental de la práctica, y gestionarlo sin valorar correcta y completamente a las personas puede afectar la atención individualizada y ocasionarles alteraciones en la integridad, seguridad y satisfacción (14).

Desde la perspectiva del sujeto de cuidado, paciente o cuidador familiar, el bienestar durante la hospitalización se relaciona con una atención técnicamente sólida, dada por quienes tienen capacitación y competencia adecuadas, y con las relaciones interpersonales caracterizadas por la información y buen trato de parte del personal de enfermería.

Para pacientes y cuidadores familiares, las necesidades psicosociales son igual de importantes a las físicas y fisiológicas, pero muchas veces menos evidentes para quienes los atienden (15). Los familiares se preocupan por el confort y soporte de sus seres queridos, y presentan necesidades de seguridad, información y proximidad 
(16); recibir educación para el cuidado constituye para ellos una prioridad (17). De igual forma, en medio de esta atención, las actividades de la vida diaria (AVD) representan el centro de su autonomía (18).

Desde la percepción del desempeño de enfermería, es preciso comprender que todos los pacientes y cuidadores familiares sanos o enfermos tienen necesidades básicas, y que es su responsabilidad buscar que estas se atiendan para procurar su adaptación durante la hospitalización. En tal sentido, la identificación de necesidades exige una valoración individual que garantice la privacidad, como base para el cuidado integral (19). Resulta, además, útil contar con un listado de necesidades básicas para revisar sobre este cuáles están satisfechas y en cuáles se requiere apoyo de enfermería (20). Un instrumento básico para la valoración y registro puede facilitar y mejorar la atención, y permite hacer visible el trabajo de enfermería (21). Con respecto a los registros, se recomienda que sean sistemáticos y que aprovechen la tecnología disponible (22). Dentro de estos, las escalas la de Barthel, que contemplan las necesidades básicas y el grado de dependencia del paciente según su estado actual, puede ser particularmente útil para orientar el trabajo de enfermería (23). En cualquier caso, sistematizar las acciones de enfermería exige contar con un perfil del servicio (24), y preparar al talento humano para la atención adecuada e integral a las necesidades básicas, mediante talleres, trabajo en grupo u otra forma de sensibilización que garantice una buena atención (25). Todos los procedimientos, aún los considerados más básicos como el baño en cama, se deben respaldar con la mejor evidencia disponible (26).

Por último, desde la perspectiva de las implicaciones de la atención a las necesidades básicas, la literatura señala que al dar valor a la participación de los pacientes y familiares, invertir en el desarrollo de las personas, el trabajo en equipo, la socialización de la información, la innovación y a la creatividad como fundamentales en la satisfacción con los cuidados, es posible tener un impacto sobre su calidad con la consecuente satisfacción de los usuarios del servicio de enfermería (27). El cuidado invisible, por el contrario, no logra este cometido (28).

La identificación de las necesidades básicas del paciente y sus familiares permite generar propuestas para mejorar la calidad del cuidado (29), y puede llevar a enfermería a proponer e implementar nuevas políticas frente al servicio, como estrategias que fortalezcan la continuidad en la asistencia, mayor tiempo o recurso de personal y mayor dedicación a la educación (30).

\section{Fase II}

El diagnóstico del nivel de adaptación durante cuatro meses, de los pacientes y sus cuidadores familiares en la atención a las necesidades básicas, señaló un nivel medio-alto en el caso del paciente, y un nivel medio-bajo en los cuidadores. Es decir, la experiencia de hospitalización es, con frecuencia, más compleja para el familiar, a quien no siempre se le considera como sujeto de cuidado. La observación de la respuesta de enfermería así lo corroboró.

\section{Fase III}

El planteamiento del subproceso "asistencia a necesidades básicas por enfermería" — validado y ajustado - orientó la transformación a momento de cuidado, e incluyó 5 etapas en las cuales se consideraron sus contenidos, actividades y herramientas de soporte (tabla 1). 
TABLA 1

Guía de enfermería para atender las necesidades básicas en pacientes hospitalizados y sus cuidadores familiares, Clínica Universidad de La Sabana

\begin{tabular}{|c|c|c|c|}
\hline Fase & Contenido & Actividad & Herramienta \\
\hline Identificación & $\begin{array}{l}\text { Valoración de las } \\
\text { necesidades del } \\
\text { paciente y su familia, } \\
\text { y priorización. }\end{array}$ & $\begin{array}{l}\text { Identifica las necesidades del paciente y su familia con } \\
\text { respecto a: } \\
\text { - Necesidades fisiológicas frente a las necesidades de sus } \\
\text { funciones de la teoría de adaptación de Roy. } \\
\text { - Identificación del entorno del paciente: solicitudes } \\
\text { especiales, aislamientos, compañía permanente, privacidad. } \\
\text { - Identificación de expectativas o el rol del paciente y } \\
\text { cuidador familiar frente el cuidado. } \\
\text { - Verifica percepción sobre la comodidad, bienestar y necesidad } \\
\text { de apoyo con el paciente y su familia. }\end{array}$ & $\begin{array}{l}\text { Bitácora } \\
\text { Escala Barthel } \\
\text { Comunicación entre el paciente y su familia con el } \\
\text { equipo de enfermería. }\end{array}$ \\
\hline Definición & $\begin{array}{l}\text { Definición del Plan } \\
\text { de atención de } \\
\text { enfermería. }\end{array}$ & $\begin{array}{l}\text { Propone un plan de cuidado y establece metas conjuntas con } \\
\text { el paciente y su cuidador familiar, e integra las propuestas con } \\
\text { el equipo interdisciplinario y las socializa. }\end{array}$ & $\begin{array}{l}\text { Nanda/ NIC/NOC. } \\
\text { Briefing. }\end{array}$ \\
\hline Atención & $\begin{array}{l}\text { Ejecución del plan de } \\
\text { atención de } \\
\text { enfermería. }\end{array}$ & $\begin{array}{l}\text { La enfermera con la participación del paciente, el cuidador } \\
\text { familiar y el personal auxiliar de enfermería, realiza la } \\
\text { asistencia a la necesidad. }\end{array}$ & Plan de Cuidados Bitácora. \\
\hline Evaluación & $\begin{array}{l}\text { Evaluación de la } \\
\text { adaptación del } \\
\text { paciente y cuidador } \\
\text { familiar. }\end{array}$ & $\begin{array}{l}\text { La enfermera verifica, con el paciente y su cuidador familiar, } \\
\text { cuál es su nivel de adaptación: } \\
\text { Evalúa el cuidado junto con el paciente y su cuidador familiar. } \\
\text { Verifica logro de metas y establece nuevas. } \\
\text { Evalúa la realización de las actividades delegadas. } \\
\text { Autoevalúa las intervenciones asumidas. }\end{array}$ & Registros de enfermería. Bitácora. \\
\hline Registro & $\begin{array}{l}\text { Registro del plan de } \\
\text { cuidado. }\end{array}$ & $\begin{array}{l}\text { La enfermera registra el plan de cuidado realizado teniendo en } \\
\text { cuenta las fases anteriores. }\end{array}$ & Registros de enfermería. Bitácora. \\
\hline
\end{tabular}

Fuente: elaboración propia (2018)

Finalmente, se encuentra la medición del subproceso de asistencia a necesidades básicas, implementado por enfermería, que tomó los datos del diagnóstico como línea de base, y se midió a partir de este durante 2 años.

Los aspectos revisados son lineamiento institucional, claramente definido del número de pacientes y familiares atendidos bajo este esquema, con respecto al número de pacientes y familiares atendidos por enfermería; los insumos requeridos para implementarlo; el nivel de satisfacción de los pacientes y de sus familiares con la atención a las necesidades básicas; y la percepción y adherencia de enfermería al subproceso. Frente a la percepción de la satisfacción de sus necesidades, participación y fortalecimiento de la autonomía, se identificaron signos de alarma; riesgos y acciones de autocuidado. Su resultado permitió adoptar de manera definitiva el subproceso de enfermería propuesto para la asistencia a necesidades básicas en la institución. A continuación, se ilustra, con un ejemplo del año 2018, la percepción de satisfacción de las necesidades del paciente y la adherencia de enfermería, en donde llama particularmente la atención la estrecha cercanía entre el comportamiento del enfermero(a), del paciente hospitalizado y su cuidador familiar (figura 1). 


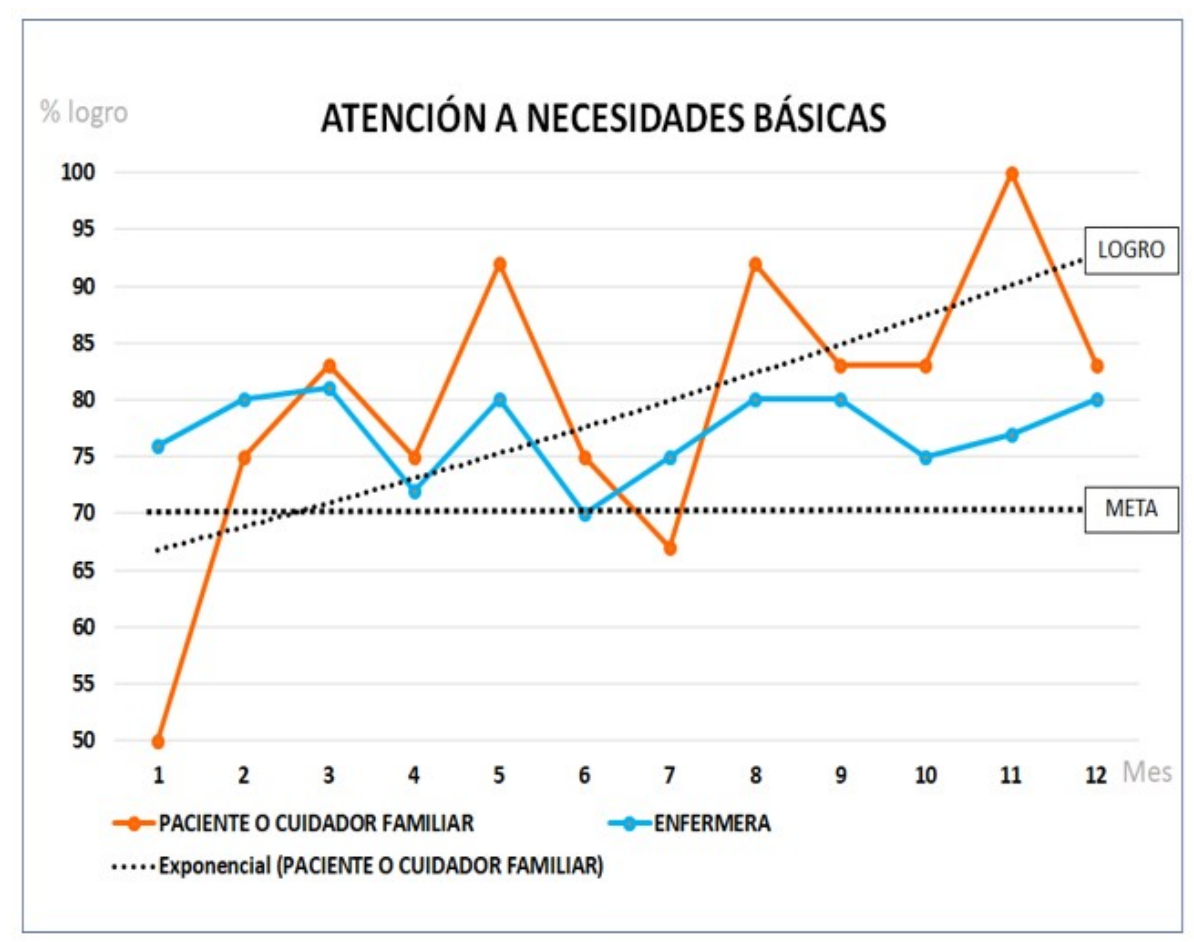

FIGURA 1.

Seguimiento de la atención de necesidades básicas en pacientes hospitalizados y sus cuidadores familiares, Clínica Universidad de La Sabana, 2018 Fuente: elaboración propia (2018)

\section{Discusión}

A pesar de considerar la atención a las necesidades como uno de los esquemas más básicos de enfermería, el lineamiento de un modelo institucional, que busca la adaptación mediante el compromiso y el tono humano (4), puede ser definitivo para lograrla. Este exige, de una parte, centrar la práctica en el paciente y su cuidador familiar, respetando su dignidad y buscando fortalecer su autonomía; y de otra, tener en cuenta la mejor evidencia disponible. Algunos autores lo han referido como "dar al cuidado un valor diferencial" (31).

Experiencias similares de transformación a partir de la aplicación de modelos de práctica profesional han sido reportadas (32), pero a diferencia de ellas, la presente ha tenido seguimiento mediante indicadores objetivos.

Para enfermería, esta propuesta logró sensibilizar frente a un enfoque holístico, como se ha recomendado (13), trasciende la mirada de las personas como seres vulnerables (12) y retoma planteamientos de cuidado para fomentar su autonomía en medio de la situación $(10,11)$, sin olvidar la importancia de contar con la mejor evidencia disponible (26), y de fortalecer el vínculo tanto con los pacientes y sus cuidadores familiares, atendiendo sus criterios de satisfacción con la atención (14). El presente desarrollo buscó, como lo sugieren experiencias anteriores, que los pacientes y sus familiares perciban que todas sus necesidades son verdaderamente escuchadas y atendidas $(15,16,17)$.

El levantamiento del subproceso incluyó la valoración como fundamento de la atención de enfermería (29), generando herramientas que atienden la experiencia del paciente y su cuidador familiar bajo el esquema de las AVD (18), y con empleo de la escala de Barthel (23). Con base en ellos, se desarrolló el listado de necesidades básicas para revisar cuáles requieren apoyo e intervención de enfermería (20,21), así como su 
sistematización (24), con apoyo de la tecnología (22). Se consideró también el seguimiento y motivación al personal de enfermería (25), estrategia fundamental para sensibilizar en el rol y el valor del cuidado.

\section{Conclusiones}

- Este proceso constituye una innovación en medio de la rutina de enfermería (27), para mejorar de forma concreta la calidad del cuidado en este campo (29) que reciben las personas a nivel institucional.

- Se buscó que este cuidado se evidencie, se cuantifique y se cualifique respondiendo a la experiencia de quienes lo reciben (30).

- Se transformó la rutina básica de enfermería conocida como "Atención a las necesidades básicas", cuando se le imprimieron dos condiciones: la primera, el compromiso, requerido para soportarlo en la mejor evidencia disponible, mejorando la calidad del cuidado; la segunda, el acercamiento humano, con respeto y reconocimiento de la dignidad de las personas, favoreciendo el vínculo y la autonomía de las mismas, sus cuidadores y el rol de la enfermería.

\section{Referencias}

1. Castro-Molina F-J. Abraham Maslow, las necesidades humanas y su relación con los cuidadores profesionales. Cult los Cuid [Internet]. 2018;22(52):102-108. https://doi.org/10.14198/cuid.2018.52.09

2. Roy, C. Nursing Knowledge in the 21st Century Domain-Derived and Basic Science Practice-Shaped. Adv Nurs Sci [Internet]. 2019;42(1): 28-42. https://doi.org/10.1097/ANS.0000000000000240

3. Polit D, Tatano C. Investigación en Enfermería: Fundamentos para el uso de la evidencia en la práctica de la enfermería. 9. ed. Barcelona: Wolters Kluwer; 2018.

4. Arroyo-Marlés LP, Guevara-Lozano M, Pérez-Giraldo B, Sánchez-Herrera B. Commitment and a sense of humanity for the adaptation of patients during hospital care. J Nurs Manag [Internet]. 2018;26(5):548-554. https://doi. org/10.1111/jonm.12581

5. Miranda-Novales MG, Villasís-Keever MA. El protocolo de investigación. Parte I. Rev Alergia Méx. 2015;62:312-317. https://doi.org/10.29262/ram.v66i1.594

6. Universidad de La Sabana. Proyecto Educativo Institucional PEI. Política ambiental Universidad de La Sabana. Fecha desconocida [citado el 10 septiembre de 2017]. Disponible en: https://www.unisabana.edu.co/fileadmin/Archivos_de_usuario/Documentos/Documentos_la_Universid ad/Docs_Institucionales/2._Proyecto_Educativo_Institucional_-PEI.pdf

7. Christmals CD, Gross JJ. An integrative literature review framework for postgraduate nursing research reviews. EJRMS. 2017;5(1):7-15. Disponible en: https://www.idpublications.org/wp-content/uploads/2016/12/Full-Paper-AN-INTEGRATIVE-LITER ATURE-REVIEW-FRAMEWORK-FOR-POSTGRADUATE-NURSING-RESEARCH-REVIEWS.pdf

8. Lobiondo G, Haber J. Nursing Research: Methods and Critical Appraisal for Evidence-Based Practice. 9. ed. San Luis (MO): Elsevier; 2018. 552 p.

9. Guevara-Lozano M, Arroyo-Marlés LP, Pérez-Giraldo B, Sánchez-Herrera B. Commitment and human tone: the difference between traditional service and nursing care. Invest. Educ. Enferm [Internet]. 2019;37(1). https://d oi.org/10.17533/udea.iee.v37n1e05

10. Armijo PP. Henderson's theory and its application in advanced nursing care in a pediatric ward. Medwave [Internet]. 2012;12(10):e5548. https://doi.org/10.5867/medwave.2012.10.5548

11. van het Bolscher-Niehuis MJT, den Ouden MEM, de Vocht HM, Francke AL. Effects of self-management support programmes on activities of daily living of older adults: A systematic review. Int J Nurs Stud [Internet]. 2016;61:230-247. https://doi.org/10.1016/j.ijnurstu.2016.06.014 
12. Esplendori GF. Reacciones adversas a la donación de sangre total, necesidades humanas básicas y diagnósticos de enfermería: una reflexión. Rev da Esc Enferm da USP [Internet]. 2018;51. https://doi.org/10.1590/s1980-22 0x2017005003284

13. Grasse AP, Bicudo SDS, Primo CC, Zucolotti C, Belonia CSF de O, Bringuente ME de O, et al. Diagnóstico e intervenciones de enfermería para la persona con úlcera venosa. Acta Paul Enferm [Internet]. 2018;31(3):280-290. https://doi.org/10.1590/1982-0194201800040

14. Carvajal-Carrascal G, Montenegro-Ramírez JD. Higiene: cuidado básico que promueve la comodidad en pacientes críticos. Enferm Global [Internet]. 2015;14:340-350. https://doi.org/10.6018/eglobal.14.4.231831

15. Teston EF, Fukumori EFC, Benedetti GM dos S, Spigolon DN, Costa MAR, Marcon SS. Sentimientos y dificultades vivenciadas por pacientes oncológicos a lo largo de los itinerarios diagnóstico y terapêutico. Esc Anna Nery [Internet]. 2018;22(4). https://doi.org/10.1590/2177-9465-EAN-2018-0017

16. Alsharari AF. The needs of family members of patients admitted to the intensive care unit. Patient Prefer Adherence [Internet]. 2019;13:465-473. https://doi.org/10.2147/PPA.S197769

17. Félix ND de C, Ramos N de M, Nascimento MNR, Moreira TMM, Oliveira CJ de. Diagnósticos de enfermería de la CIPE ${ }^{\circledR}$ para personas con síndrome metabólica. Rev Bras Enferm [Internet]. 2018;71(supl. 1):467-474. ht tps://doi.org/10.5205/1981-8963-v12i12a238081p3181-3189-2018

18. Candela F, Zucchetti G, Rabaglietti E, Magistro D, Ortega E. Preventing loss of basic activities of daily living and instrumental activities of daily living in elderly: Identification of individual risk factors in a holistic perspective. Holist Nurs Pract. 2015;29(5):313-322. https://doi.org/10.1097/HNP.0000000000000106

19. Alba de la Torre D, Moreda Díaz-Pavón M, Fernández-Camacho R, Fernández-Rodríguez M, Barahona Muñoz M. Percepción de necesidades básicas: ¿es necesaria una consulta de enfermería para pacientes en hemodiálisis? Enfermería Nefrológica [Internet]. 2013;16(supl. 1):93-94 https://doi.org/10.4321/S2254-28842013000500 046

20. Oliveira PB de, Tavares DM dos S. Condiciones de salud de ancianos residentes en Institución de Larga Permanencia según necesidades humanas básicas. Rev Bras Enferm [Internet]. 2014;67(2):241-246. https://doi.org/10.5935 /0034-7167.20140032

21. Marques DKA, Silva K de L, Nóbrega MML da. Escolares hospitalizados: proposición de un instrumento para recolección de datos a la luz de la teoría de Horta. Rev Gaúcha Enferm [Internet]. 2017;37(espe). https://doi.o $\mathrm{rg} / 10.1590 / 1983-1447.2016 . e s p .2016-0038$

22. Errico L de SP de, Bicalho PG, Oliveira TCFL de, Martins EF. El trabajo del enfermero en el prenatal de alto riesgo bajo la óptica de las necesidades humanas básicas. Rev Bras Enferm [Internet]. 2018;71(supl. 3):1257-1264. ht tps://doi.org/10.1590/0034-7167-2017-0328

23. Liu W, Unick J, Galik E, Resnick B. Barthel Index of Activities of Daily Living Item Response Theory Analysis of Ratings for Long-Term Care Residents. Nurs Res [Internet]. 2015;64(2):88-99. https://doi.org/10.1097/NN R.0000000000000072

24. Souza Neto VL de, Andrade LL de, Agra G, Costa MML, Silva RAR da. Perfil diagnóstico de enfermería de los pacientes hospitalizados en la unidad de enfermedades infecciosas. Rev Gaúcha Enferm [Internet]. 2015;36(3):79-85. https://doi.org/10.1590/1983-1447.2015.03.51495

25. Urure-Velazco IN, Campos-Soto R, Ventura-Miranda CT, Navarro-Quintanilla T. Relaciones humanas entre el profesional de enfermería y paciente en la satisfacción de las necesidades en el servicio de cirugía del Hospital Santa María del Socorro Ica, setiembre 2008-agosto 2009. Rev. Enferm. Vanguard [Internet]. 2016;4(1):24-29. https://doi.org/10.35563/revan.v4i1.235

26. Flórez-Ramos JM, González-Consuegra RV, Guerra-Urrego KA. Modelo de adaptación de Roy en el baño en cama. Av en Enfermería [Internet]. 2016;34(3):215-225. https://doi.org/10.15446/av.enferm.v34n3.48780

27. Freitas JS de, Silva AEB de C, Minamisava R, Bezerra ALQ, Sousa MRG de. Calidad de los cuidados de enfermería y satisfacción del paciente atendido en un hospital de enseñanza. Rev Lat Am Enfermagem [Internet]. 2014;22(3):454-460. https://doi.org/10.1590/0104-1169.3241.2437 
28. Lopera-Arango. Cuidar al paciente sin estar con él: invisibilidad del cuidado de enfermería en servicios de hospitalización. Investigación y Educación en Enfermería [Internet]. 2018;36(3). Disponible en: http://www.s cielo.org.co/scielo.php?script $=$ sci_arttext\&pid=S0120-53072018000300010\&lng=en\&nrm=iso\&tlng=es

29. Martins PF, Perroca MG. Care necessities: the view of the patient and nursing team. Rev Bras Enferm [Internet]. 2017;70(5):1026-1032. https://doi.org/10.1590/0034-7167-2016-0197

30. Zaken ZB, Maoz E, Raizma E. Needs of Relatives of Surgical Patients: Perceptions of Relatives and Medical Staff. Medsurg Nurs [Internet]. 2018;27(2):110-116. Disponible en: https://search-ebscohost-com.ez.unisabana.edu $. c o /$ login. aspx $?$ direct $=$ true\&AuthType $=\mathrm{ip} \& \mathrm{db}=\mathrm{a} 9 \mathrm{~h} \& \mathrm{AN}=129092744 \&$ lang=es\&site=eds-live\&scope $=$ site

31. Waldow VR. Cuidado humano: la vulnerabilidad del ser enfermo y su dimensión de trascendencia. Index Enferm [Internet]. 2014;23(4):234-238. https://doi.org/10.4321/S1132-12962014000300009

32. Carvajal-Hermida EY, Sánchez-Herrera B. "Nursing Care with a Human Approach": A Model for Practice with Service Excellence. Aquichán [Internet]. 2018;18(2):149-159. https://doi.org/10.5294/aqui.2018.18.2.3

\section{Notas}

* Artículo de investigación

Licencia Creative Commons CC BY 4.0

Cómo citar este artículo: Arroyo Marlés LP, Pérez Giraldo B, Nonsoque Cholo MA, Sánchez Herrera B, Guevara Lozano M. Transformar la asistencia de necesidad básica en un momento de cuidado. Investig Enferm Imagen Desarr. 2020;22. https://doi.org/10.11144/Javeriana.ie22.tanb 